\title{
Chest magnetic resonance imaging: a protocol suggestion*
}

\author{
Ressonância magnética do tórax: sugestão de protocolo
}

\section{Bruno Hochhegger $^{1}$, Vinícius Valério Silveira de Souza ${ }^{2}$, Edson Marchiori ${ }^{3}$, Klaus Loureiro Irion ${ }^{4}$, Arthur Soares Souza Jr. ${ }^{5}$, Jorge Elias Junior ${ }^{6}$, Rosana Souza Rodrigues ${ }^{7}$, Miriam Menna Barreto ${ }^{7}$, Dante Luiz Escuissato $^{8}$, Alexandre Dias Mançano ${ }^{9}$, César Augusto Araujo Neto ${ }^{10}$, Marcos Duarte Guimarães ${ }^{11}$, Carlos Schuler Nin $^{2}$, Marcel Koenigkam Santos ${ }^{12}$, Jorge Luiz Pereira e Silva ${ }^{13}$}

Hochhegger B, Souza VVS, Marchiori E, Irion KL, Souza Jr AS, Elias Junior J, Rodrigues RS, Barreto MM, Escuissato DL, Mançano AD, Araujo Neto CA, Guimarães MD, Nin CS, Koenigkam-Santos M, Pereira e Silva JL. Chest magnetic resonance imaging: a protocol suggestion. Radiol Bras. 2015 Nov/ Dez;48(6):373-380.

Abstract In the recent years, with the development of ultrafast sequences, magnetic resonance imaging (MRI) has been established as a valuable diagnostic modality in body imaging. Because of improvements in speed and image quality, MRI is now ready for routine clinical use also in the study of pulmonary diseases. The main advantage of MRI of the lungs is its unique combination of morphological and functional assessment in a single imaging session. In this article, the authors review most technical aspects and suggest a protocol for performing chest MRI. The authors also describe the three major clinical indications for MRI of the lungs: staging of lung tumors; evaluation of pulmonary vascular diseases; and investigation of pulmonary abnormalities in patients who should not be exposed to radiation.

Keywords: Magnetic resonance imaging; Lung; Chest; Protocol; Sequences.

Resu mo Nos últimos anos, com o desenvolvimento de sequências ultrarrápidas, a ressonância magnética (RM) tem-se estabelecido como uma ferramenta de diagnóstico por imagem de grande valor. Em virtude dos aperfeiçoamentos na velocidade de aquisição e na qualidade das imagens, a RM é atualmente um método apropriado também para o estudo de doenças pulmonares. A principal vantagem da RM é sua combinação única que permite avaliação morfológica e funcional em um mesmo exame de imagem. Neste artigo iremos revisar aspectos técnicos e sugerir um protocolo para a realização de RM do tórax. Também serão descritas as três maiores indicações de RM do tórax: estadiamento para neoplasia pulmonar, avaliação de doença vascular do pulmão e investigação de doenças pulmonares em pacientes que não devem ser expostos à radiação ionizante.

Unitermos: Ressonância magnética; Pulmão; Tórax; Protocolo; Sequências.

* Study developed at Irmandade Santa Casa de Misericórdia de Porto Alegre, Porto Alegre, RS, Brazil.

1. PhD, Associate Professor, Universidade Federal de Ciências da Saúde de Porto Alegre (UFCSPA), Porto Alegre, RS, Brazil.

2. MD, Resident in Radiology and Imaging Diagnosis, Irmandade Santa Casa de Misericórdia de Porto Alegre, Porto Alegre, RS, Brazil.

3. PhD, Full Professor Emeritus, Universidade Federal Fluminense (UFF), Niterói, RJ, Brazil.

4. PhD, Consultant Radiologist, Liverpool Heart and Chest Hospital NHS Trust, Liverpool, UK.

5. PhD, Professor, Faculdade de Medicina de São José do Rio Preto (Famerp), São José do Rio Preto, SP, Brazil.

6. PhD, Associate Professor, Centro de Ciências das Imagens e Física Médica (CCIFM) - Faculdade de Medicina de Ribeirão Preto da Universidade de São Paulo (FMRP-USP), Ribeirão Preto, SP, Brazil.

7. PhD, Professor, Program of Post-graduation in Radiology, Universidade Federal do Rio de Janeiro (UFRJ), Rio de Janeiro, RJ, Brazil.

8. PhD, Associate Professor, Department of Medical Practice, Universidade Federal do Paraná (UFPR), Curitiba, PR, Brazil.

9. MD, Radiologist, Radiologia Anchieta, Taguatinga, DF, Brazil.

10. PhD, Associate Professor, Universidade Federal da Bahia (UFBA), Salvador, BA, Brazil.

11. PhD, Professor, Program of Post-graduation stricto sensu, A.C.Camargo Cancer Center, São Paulo, SP, Brazil.

12. PhD, Attending Physician at Hospital das Clínicas da Faculdade de Medicina de Ribeirão Preto da Universidade de São Paulo (HCFMRP-USP), Ribeirão Preto, SP, Brazil.

13. PhD, Associate Professor, Department of Medicine and Diagnostic Support, Universidade Federal da Bahia (UFBA), Salvador, BA, Brazil.

\section{INTRODUCTION}

Pulmonary parenchyma imaging represents a unique challenge for magnetic resonance imaging (MRI). Limited signal intensity is caused by low proton density, susceptibility artifacts are due to differences between tissue and air, besides physiological motion (cardiac pulsation, respiration). Recently, further improvements in MRI techniques have widened the potential for investigation of pulmonary parenchymal diseases. Such techniques include very short echo times, ultrafast turbo-spin-echo acquisitions, projection reconstruction technique, breathhold imaging, and recently developed contrast agents (for perfusion and ventilation imaging $)^{(1-3)}$.

In healthy lungs, the tissue density is $0.1 \mathrm{~g} / \mathrm{cm}^{3}$, which is about tenfold lower than in other soft tissue organs. As the MRI signal intensity is directly proportional to the tissue proton density, even under perfect imaging conditions (i.e. neglecting relaxation effects), the MRI signal from the

Mailing Address: Dr. Vinícius Valério Silveira de Souza. Rua José do Patrocínio, 111, ap. 12, Cidade Baixa. Porto Alegre, RS, Brazil, 90050-001. E-mail: viniciusvss@ gmail.com.

Received March 11, 2014. Accepted after revision July 28, 2014. 
lung is ten-times weaker than that from adjacent tissues. The low signal-to-noise ratio makes proton MRI of the lung microstructure challenging. Signal averaging can be employed to increase the signal-to-noise ratio, but this extends the image acquisition times beyond $10 \mathrm{~min}$ per data set, which would make the protocols unsuitable for clinical routine. The signal-to-noise ratio may be increased with larger voxel sizes; however, smaller lesions such as peripheral lung metastases might not be visible due to partial volume effects ${ }^{(1-3)}$.

To obtain broad clinical acceptance, MRI of the lung has to be practical, robust, and reproducible. On top of these workflow aspects, MRI should provide consistently high image quality as well as diagnostic accuracy and a positive therapeutic impact. Different scanner manufacturers already provide the necessary sequences to perform MRI of the lung $^{(1-3)}$. In the present article, the authors review the clinical and technical aspects of the method and suggest a protocol to be used in clinical routine for chest MRI.

\section{CLINICAL INDICATIONS FOR MRI OF THE LUNG}

\section{Detection and characterization of pulmonary nodules}

A recent meta-analysis reported that dynamic computed tomography (CT) and MRI, both of which are noninvasive methods, are equally accurate in distinguishing between malignant and benign solitary pulmonary nodules, and the differences between the two methods are insignificant ${ }^{(4)}$. The authors of the meta-analysis have found that, for the $10 \mathrm{dy}$ namic CT studies, MRI had a pooled sensitivity of $93 \%(95 \%$ CI: $0.88-0.97)$ and a pooled specificity of $76 \%$ (95\% CI: $0.68-0.97)^{(5)}$. Koyama et al. ${ }^{(4)}$ have reported that non-contrast-enhanced MRI of the lung is as efficient as is thin-section multidetector CT in detecting malignant nodules. The authors have also found that the overall nodule detection rate at each MRI sequence ( $82.5 \%$ ) was significantly lower than was that for multidetector CT (97.0\%), although there was no significant difference between the two techniques in terms of malignant nodules detection rate. Also, chemical shift MRI has been described to detect fat in pulmonary hamartomas $^{(5)}$ (Figure 1). Diffusion-weighted MRI may be able to be used in place of ${ }^{18} \mathrm{~F}$-fluorodeoxyglucose positron emission tomography (FDG-PET) to distinguish malignant from benign pulmonary nodules/masses with fewer false-positive results as compared with FDG-PET ${ }^{(6)}$.

\section{Tumor-node-metastasis staging}

In the tumor-node-metastasis (TNM) staging system, the T stage (tumor size and invasion degree) is the primary determinant of the neoplasia severity ${ }^{(7)}$. MRI is superior to CT for depicting the pericardium, heart, and mediastinal vessels and, therefore, it can be indicated in specific situations such as superior vena cava obstruction, myocardial invasion, or tumor spread into the left atrium via pulmonary veins ${ }^{(8)}$. In addition, MRI allows for lung cancer to be distinguished from secondary changes due to atelectasis or pneumonitis ${ }^{(7)}$. At T2-weighted images, post-obstructive atelectasis and pneu- monitis often show higher signal intensity when compared with the central tumor ${ }^{(7)}$. Ohno et al. ${ }^{(9)}$ have conducted a prospective study of 115 consecutive lung cancer patients submitted to CT, short-tau inversion-recovery turbo spinecho (STIR-TSE) imaging (Figure 2), and FDG-PET/CT, as well as surgical and pathological examination. The authors have found that, on a per-patient basis, the quantitative sensitivity and accuracy of STIR-TSE imaging $(90.1 \%$ and $92.2 \%$, respectively) were significantly higher than were the quantitative sensitivity, qualitative sensitivity, quantitative accuracy, and qualitative accuracy of co-registered FDGPET/CT (76.7\%, $74.4 \%, 83.5 \%$, and $82.6 \%$, respectively). Previous studies have demonstrated that whole-body MRI provides acceptable accuracy, and that its efficacy in lung cancer staging is comparable to that of PET/CT ${ }^{(8,9)}$. Each of those two imaging modalities has been shown to have its advantages $^{(7)}$, whole-body MRI being superior in the detection of brain and liver metastases, while PET/CT performs better for detecting lymph node and soft-tissue metastases. Diffusion-weighted MRI is a promising technique to evaluate areas that have been submitted to radiation therapy ${ }^{(7)}$. Whole-body diffusion-weighted MRI can be used for the assessment of the M (metastasis) stage in non-small cell lung cancer patients and has been shown to be as accurate as is $\mathrm{PET} / \mathrm{CT}^{(9)}$.

\section{Pulmonary thromboembolic disease}

Pulmonary embolism is the third most common cause of acute cardiovascular disease (after myocardial infarction and stroke). It frequently goes undetected and is therefore responsible for thousands of deaths every year ${ }^{(10)}$. In 2003 , Stein et al. ${ }^{(11)}$ conducted a meta-analysis of the use of gadolinium-enhanced MRI to depict acute pulmonary embolism. The authors used conventional pulmonary angiography as the reference standard. They found that the reported procedure sensitivity covered a broad range (77-100\%) and that the reported specificity was uniformly high $(95-98 \%)^{(11)}$ (Figure 3). In the most recent of the studies evaluated in this meta-analysis, Oudkerk et al. ${ }^{(12)}$ showed that the sensitivity of contrast-enhanced MRI for pulmonary embolism was $100 \%$ in central and lobar arteries, $84 \%$ in segmental arteries, and only $40 \%$ in subsegmental branches. Overall, the combined MRI protocol has been found to be more reliable and sensitive than is 16-slice multidetector $\mathrm{CT}^{(13)}$. The average MRI examination time is reported to be approximately $10 \min ^{(13)}$.

\section{Pulmonary hypertension}

The use of MRI allows a comprehensive assessment of pulmonary hypertension; especially when performing MR angiography and perfusion MRI, it is possible to differentiate between chronic thromboembolic pulmonary hypertension and pulmonary arterial hypertension ${ }^{(14,15)}$. In addition, MR angiography allows for an in-depth evaluation of the thromboembolic material location, and, for surgical planning, 

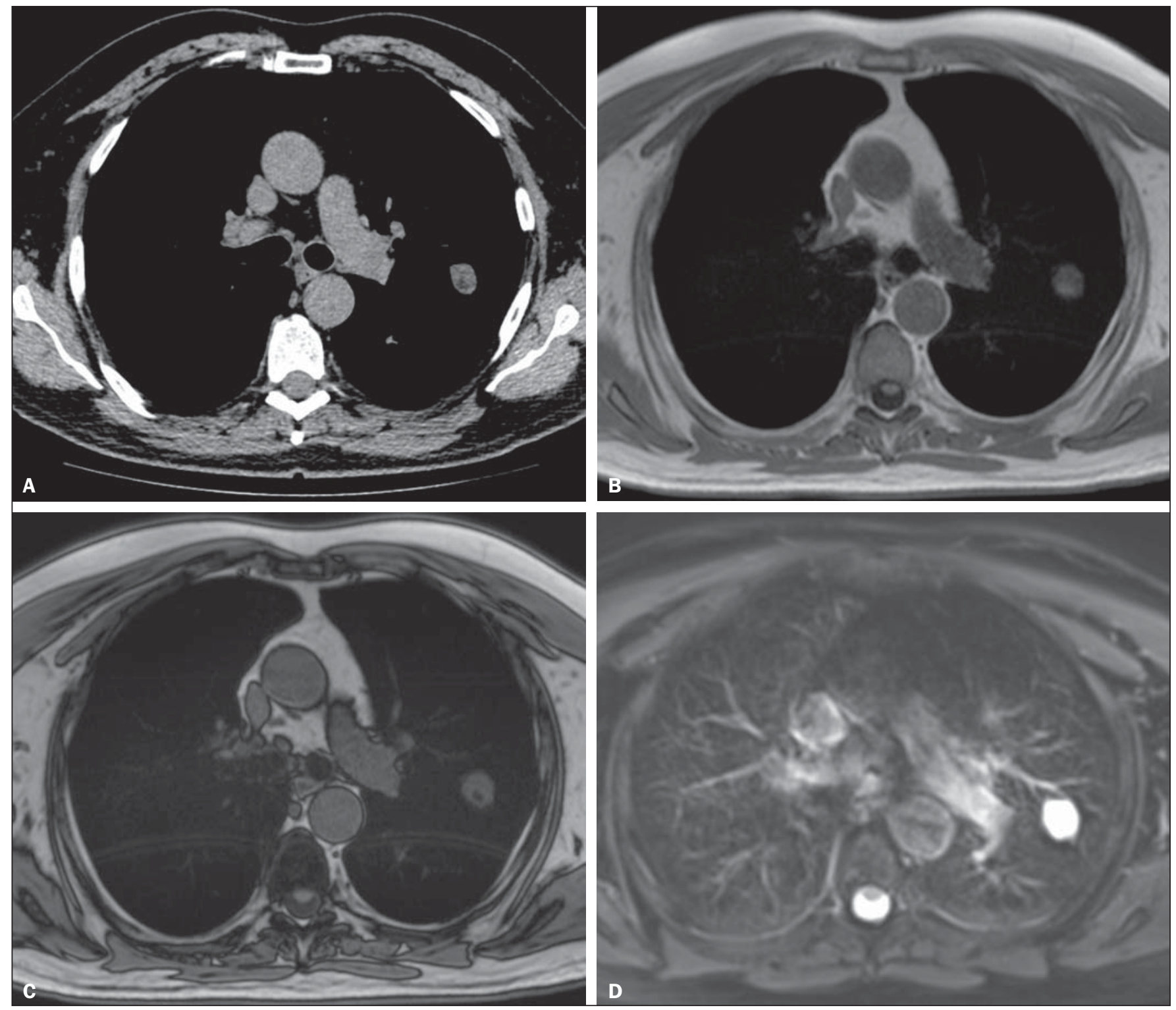

Figure 1. A 56-year-old patient with pancreatic cancer. A: Axial CT image showing low attenuation areas inside the nodule. This nodule had mean -33 HU attenuation. In-phase (B) and opposed-phase (C) images showing signal loss in the nodule, suggesting hamartoma. D: T2-weighted sequence showing high signal intensity of the nodule.
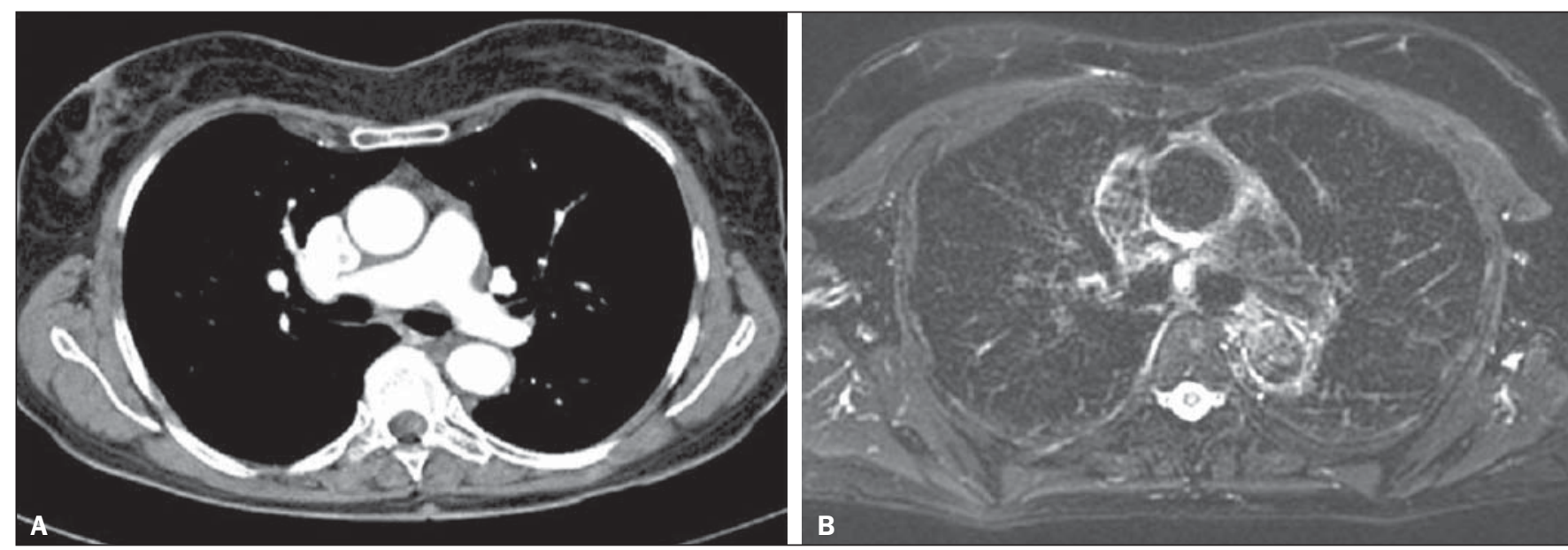

Figure 2. A: Axial CT image showing a $8 \mathrm{~mm}$ lymph node in the subcarinal station. B: Axial T2-weighted image with fat saturation showing a high signal intensity in this lymph node, suggesting metastatic disease. 


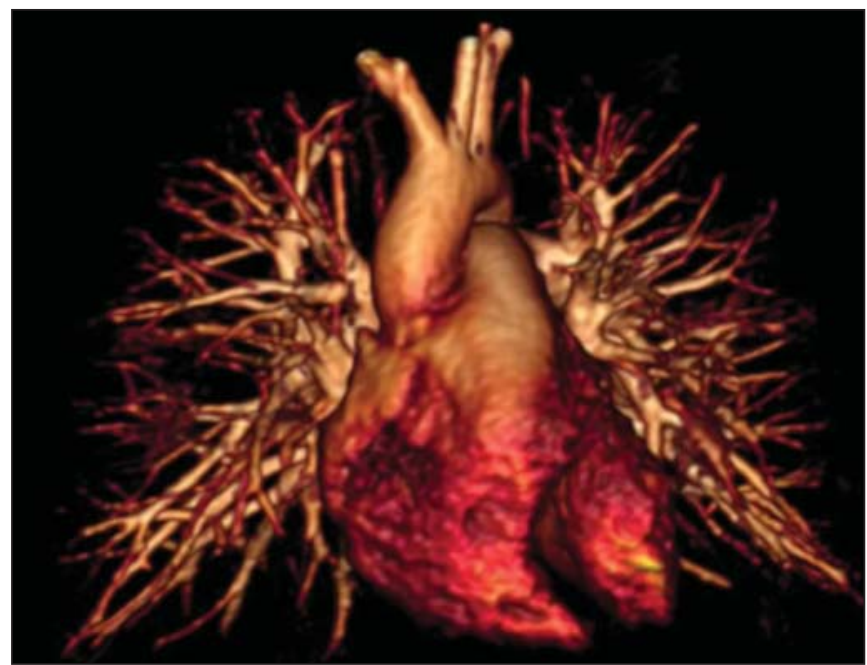

Figure 3. 3D volume rendering of MR angiography showing subsegmental resolution.

is equally as useful as are digital subtraction angiography and CT angiography ${ }^{(16,17)}$. In MRI, perfusion sequences can be quantitatively evaluated, allowing for assessing the severity of small-vessel disease. Structural imaging of the lung will allow ruling out parenchymal diseases. Measurements of blood flow and right heart pressure allow pulmonary arterial pressure and cardiac strain to be estimated, besides facilitating the identification of concomitant valvular disease ${ }^{(17)}$.

\section{Patients with cystic fibrosis}

The standard radiological tools for monitoring lung disease in patients who have cystic fibrosis are chest X-ray and high resolution CT (HRCT), and for this purpose dif- ferent scoring systems are proposed ${ }^{(18)}$. Scans obtained using thin sections provide images of lung structure with micrometer-scale resolution, and HRCT and/or MRI findings can also constitute useful outcome measures for studies of lung disease in cystic fibrosis patients ${ }^{(19,20)}$. Additionally, MRI can be used to assess various aspects of pulmonary function, including lung perfusion (Figure 4$)^{(21,22)}$, blood flow $^{(23)}$, respiratory mechanics ${ }^{(24,25)}$, and (with the administration of inhaled contrast agents) pulmonary ventilation $^{(26)}$. Recent studies have shown that MRI is highly capable of revealing the bronchiectasis typical of cystic fibrosis, as well as mucus plugging, and that MRI has a diagnostic value equal to that of CT in grading the severity of the disease with the Bhalla or Helbich score ${ }^{(27)}$.

\section{Patients with pneumonia}

The various features of pneumonia, such as ill-defined nodules, ground-glass opacities, and consolidations can be easily detected and differentiated by MRI (Figure 5). At chest MRI, extremely small opacities and calcifications represent great challenges because of the thicker slices and the low signal intensity. As a follow-up tool, MRI is recommended over CT, in order to avoid excessive exposure to ionizing radiation. The sensitivity of $\mathrm{T} 2$-weighted sequences and the potential of contrast-enhanced T1-weighted sequences can greatly facilitate the differential diagnosis ${ }^{(28)}$. In addition, incipient complications such as pericardial effusion, pleural effusion, empyema, and lung abscess are easily recognized on MRI scans ${ }^{(28)}$. In immunocompromised patients, MRI is nearly as accurate as is CT for detecting pulmonary abnormalities associated with infection ${ }^{(29-31)}$.

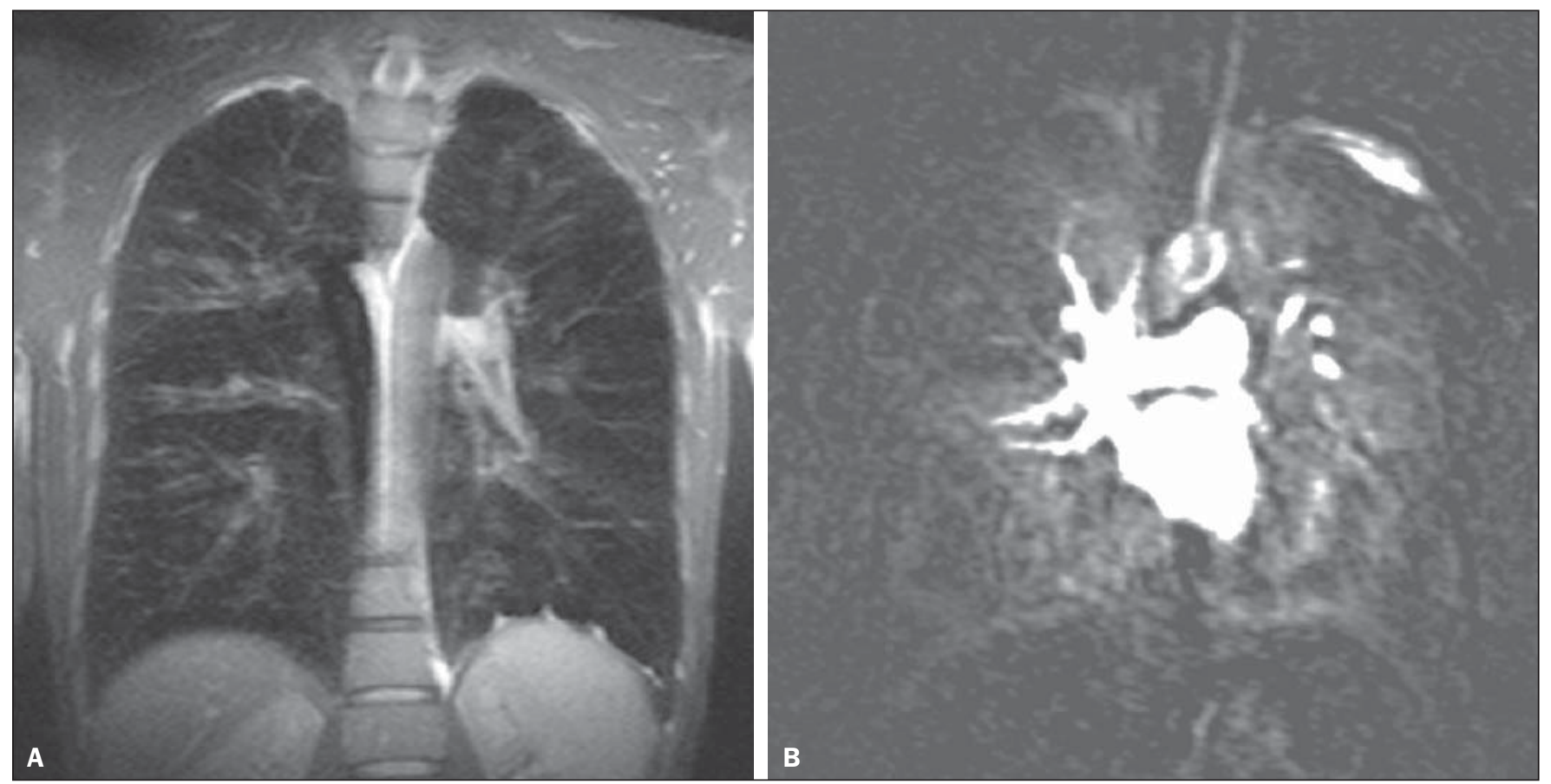

Figure 4. 32 year-old patient with cystic fibrosis. A: Coronal T1-weighted gradient echo sequence (VIBE) with 2 mm slice thickness. Note the presence of bronchiectasis with mucoid impaction. B: Pulmonary perfusion shows multiple perfusion defects better characterizing the disease severity. 
Figure 5. A: Axial HRCT image showing homogeneous, segmental ground glass opacities in the pulmonary cortex. B: Axial T2-weighted image clearly demonstrates the lesion.

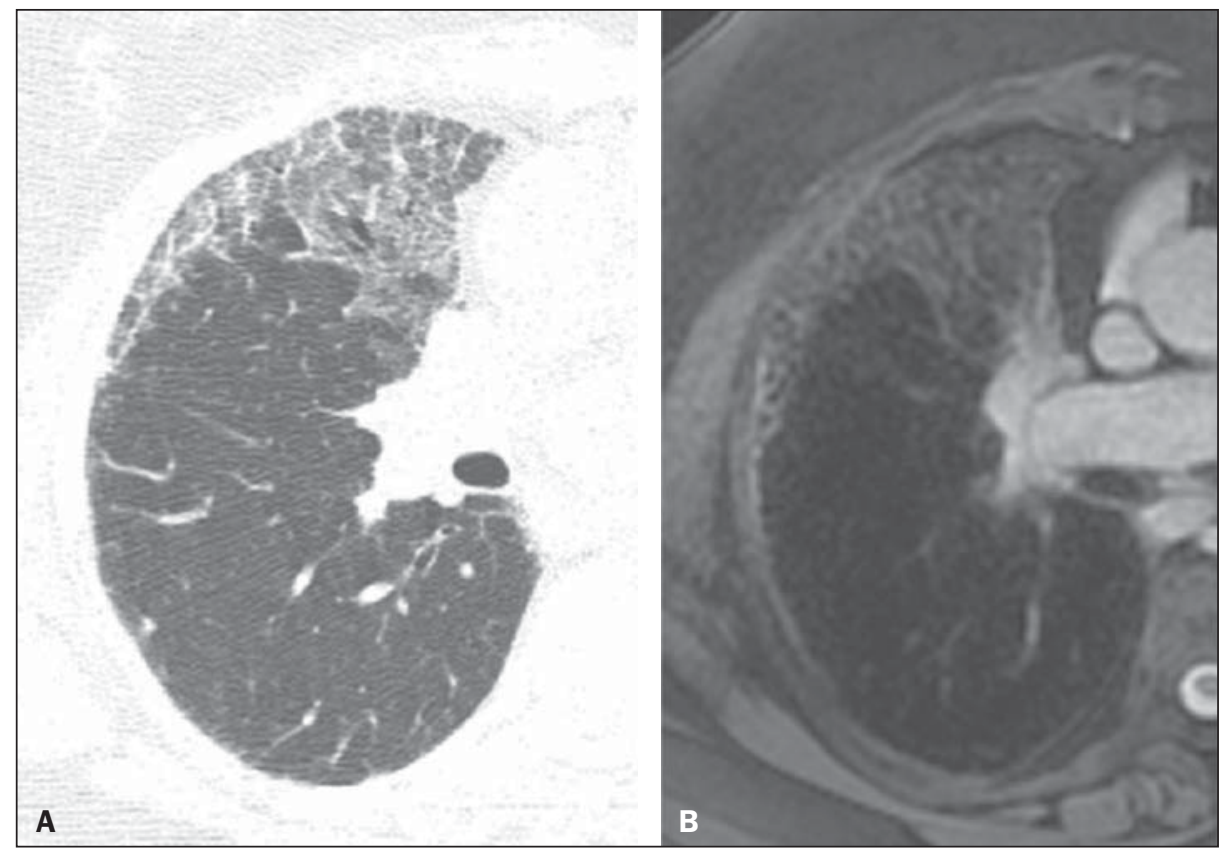

\section{TECHNICAL ASPECTS AND PROTOCOL SUGGESTION}

For MRI of the lung, standard scanners with field strength of 1.5 tesla $(\mathrm{T})$ and full parallel imaging capabilities are recommended ${ }^{(2,3,32)}$. Although higher field strength, i.e., $3 \mathrm{~T}$, will theoretically increase the signal-to-noise ratio, faster signal decay caused by susceptibility artifacts poses additional obstacles to lung imaging. Special efforts are needed to obtain similar results at $3 \mathrm{~T}$ as compared with $1.5 \mathrm{~T}$, e.g., when imaging nodules ${ }^{(33)}$

Oxygen in air is paramagnetic, and tissue is diamagnetic, which leads to a bulk magnetic susceptibility difference $(\Delta \chi$ $=8 \mathrm{ppm}$ ) at lung-air interfaces. At each tissue interface, the susceptibility difference forms a static local field gradient. The multiple microscopic surfaces presented by the airways and alveoli in the lungs thus create highly inhomogeneous local magnetic field gradients on a spatial scale smaller than the size of a typical imaging voxel $(2-5 \mathrm{~mm})^{(1-3)}$. These microscopic field gradients lead to a rapid dephasing in gra- dient echo imaging; this signal decay is typically described by an apparent transverse relaxation time $\mathrm{T} 2{ }^{*}$, which can be as short as $2 \mathrm{~ms}$ or less at $\mathrm{B} 0=1.5 \mathrm{~T}$. Thus, gradient echo MRI of lung parenchyma becomes highly challenging and requires pulse sequences with short echo times (TE $<1-2$ $\mathrm{ms})^{(1-3)}$. As the magnetic field inhomogeneity increases with B0, even shorter $\mathrm{T} 2 *$ of about $0.5 \mathrm{~ms}$ are found at $3 \mathrm{~T}$. Frequently, the expected signal-to-nose ratio gain of $3 \mathrm{~T}$ over $1.5 \mathrm{~T}$ cannot be realized, because it requires that TE is shortened accordingly. With identical pulse sequences, a shorter TE can only be achieved if more powerful gradient systems are used, but current $3 \mathrm{~T}$ MRI systems often utilize the same gradient units as high-end $1.5 \mathrm{~T}$ systems.

A basic protocol is mainly based on non-contrast breathhold sequences and free breathing diffusion-weighted imaging (Table 1). During this time, three-dimensional (3D) T1weighted gradient echo and T2-weighted fast spin echo as well as STIR sequences can be used. Respiratory, vascular and cardiac motion can be addressed by fast imaging, gating, and triggering techniques, respectively (Figure $6-\mathrm{A}-\mathrm{F})^{(34)}$

Table 1-Basic protocol.

\begin{tabular}{|c|c|c|}
\hline GE & Siemens & Slice thickness \\
\hline Axial, T2-weighted sequence FSE Fiesta & T2-weighted sequence FSE TrueFisp & $5.0 \mathrm{~mm}$ \\
\hline Coronal, T2-weighted sequence FSE Fiesta & T2-weighted sequence FSE TrueFisp & $5.0 \mathrm{~mm}$ \\
\hline Axial, T2-weighted sequence FSE with fat suppression & Axial, T2-weighted sequence Blade axial & $5.0 \mathrm{~mm}$ \\
\hline Axial diffusion-weighted & Axial diffusion-weighted & $\begin{array}{c}5.0 \mathrm{~mm} \\
\mathrm{BO} \text { and B600 }\end{array}$ \\
\hline T1-weighted sequence LAVA with fat suppression & T1-weighted sequence VIBE with fat suppression & $2.5 \mathrm{~mm}$ \\
\hline T1-weighted sequence LAVA without fat suppression & T1-weighted sequence VIBE without fat suppression & $2.5 \mathrm{~mm}$ \\
\hline T1-weighted sequence in- and out-of-phase & T1-weighted sequence in- and out-of-phase & $5.0 \mathrm{~mm}$ \\
\hline Coronal, T2-weighted sequence HASTE & T2-weighted sequence HASTE coronal & $5.0 \mathrm{~mm}$ \\
\hline Perfusion MRI T1-weighted sequence & Perfusion MRI T1-weighted sequence & 20-40 acquisition phases \\
\hline T1-weighted sequence LAVA with fat suppression & T1-weighted sequence VIBE with fat suppression & $60 \mathrm{~s}$ after injection \\
\hline
\end{tabular}




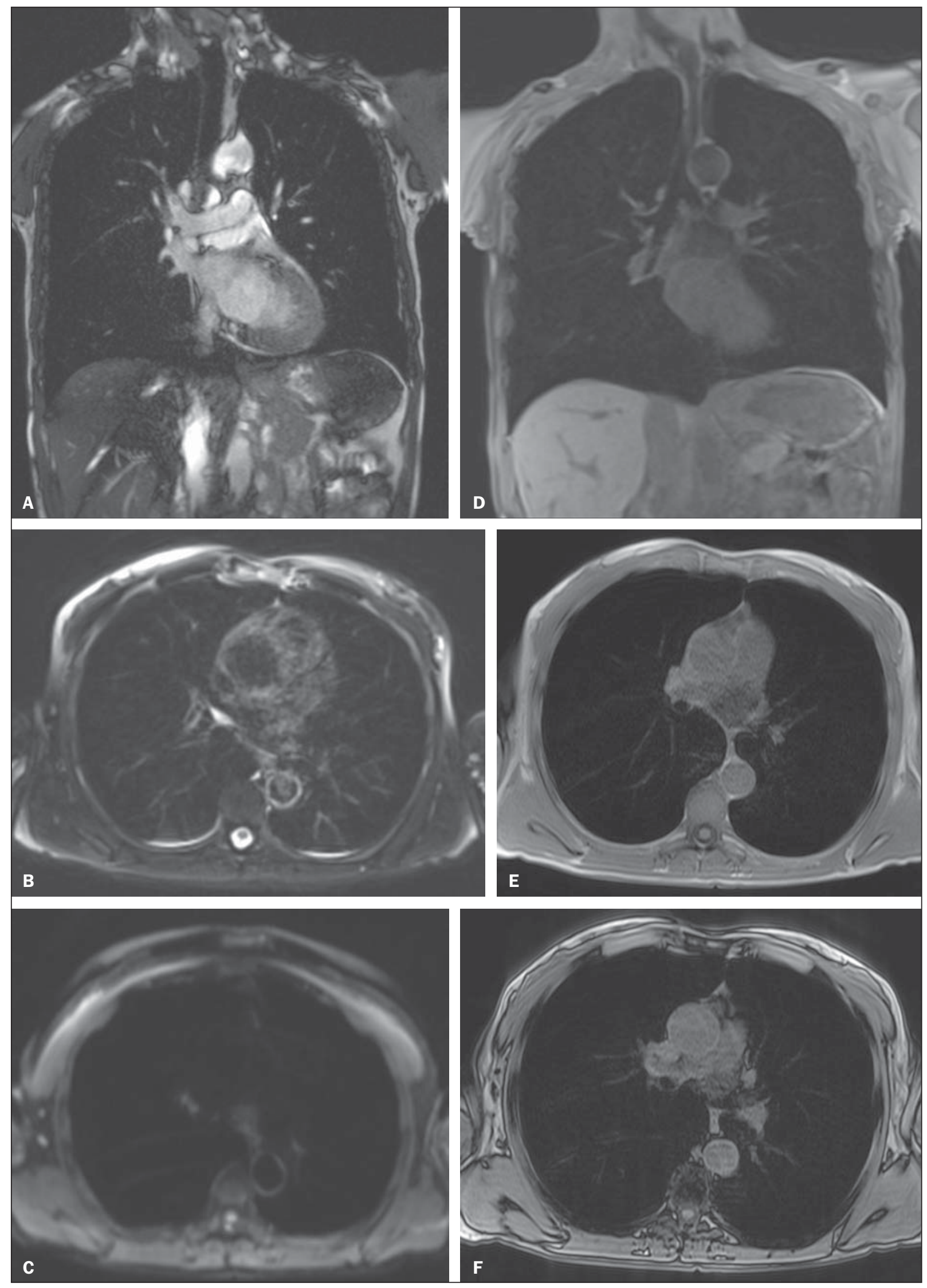

Figure 6 (A-F). Image examples for the suggested protocol. A: Coronal T2-weighted FSE TrueFisp sequence (4 mm). B: Axial, T2-weighted sequence Blade axial (4 mm). C: Axial, diffusion-weighted image ( $5 \mathrm{~mm})$. D: Coronal, T1-weighted sequence VIBE with fat suppression (4 mm). E,F: T1-weighted sequence in- and out-of-phase. 


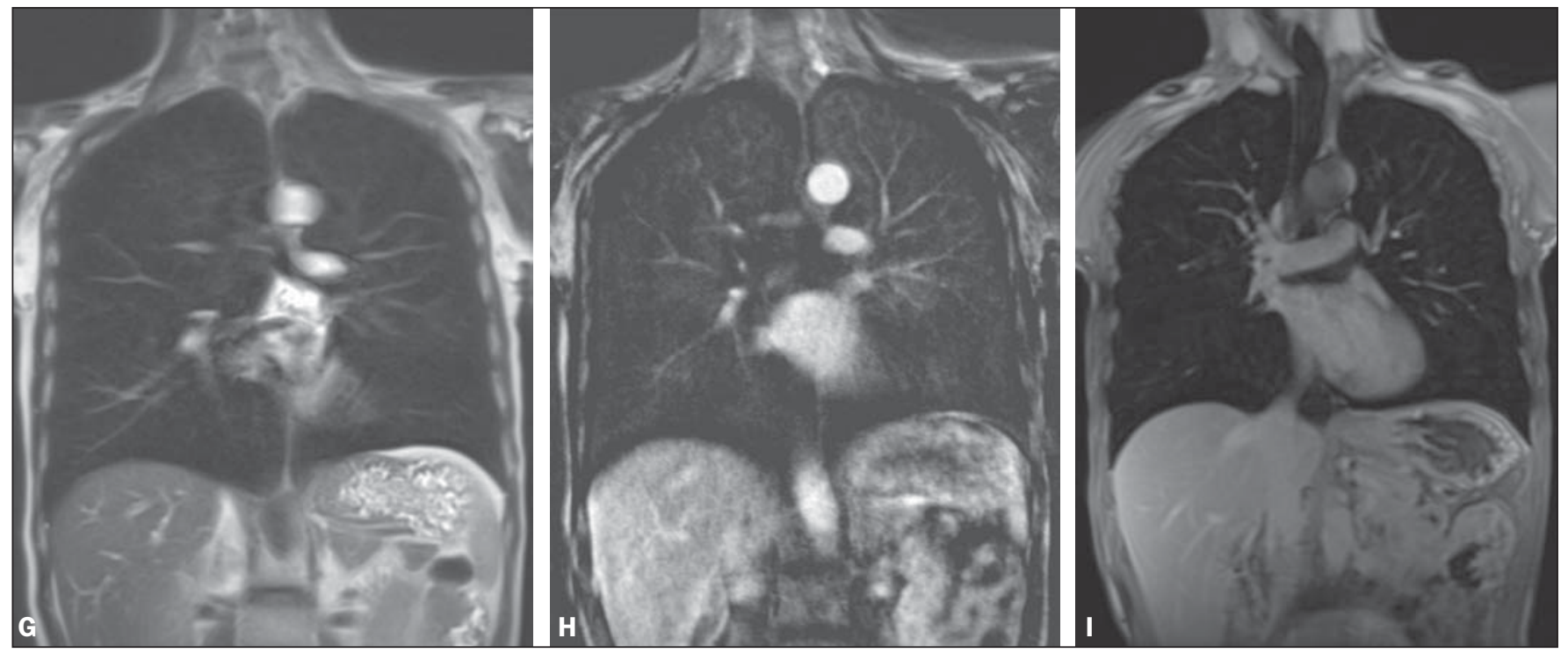

Figure 6 (G-I): Image examples for the suggested protocol. G: Coronal, T2-weighted HASTE sequence. H: Coronal, T1-weighted perfusion sequence. I: T1-weighted sequence VIBE with fat suppression.

Half-Fourier acquisition and ultra-short echo times are recommended $^{(32)}$. The basic protocol should be extended to contrast-enhanced imaging with high spatial resolution (single-phase MR angiography) or high temporal resolution as in time-resolved perfusion imaging (Figure $6-\mathrm{G}, \mathrm{H}$ ). Complicated and time-consuming sequences requiring respiratory or cardiac gating should be reserved for specific clinical scenarios. T1-weighted 3D gradient echo sequences such as a volume interpolated breath-hold examination (VIBE) are recommended for the assessment of the mediastinum, pulmonary nodules, masses and consolidations, and should be repeated with fat saturation after the administration of contrast material. In chronic obstructive pulmonary disease, for example, the contrast agent compensates for the decreased signal intensity due to the properties of the "minus-pathology"(32) (Figure 6-I). A T2-weighted fast spin echo halfFourier acquisition sequence will easily visualize pulmonary infiltrates, inflammatory bronchial wall thickening, as well as mucus and fluid accumulation. Experimental work has shown that the sensitivity of T2-weighted breath-hold or respiratory-gated sequences for infiltrates at least equals that of chest $\mathrm{X}$-ray and multidetector $\mathrm{CT}^{(13)}$.

The use of diffusion-weighted sequences in the assessment of masses or lymph node involvement is still subject to evaluation, but has also shown promising results for wholebody staging of lung cancer ${ }^{(34-36)}$. However, whole-body MRI with modern fast techniques has been suggested as a diagnostic tool for M staging, which can be implemented into a comprehensive lung cancer staging protocol. Such a technique has already proved to be sensitive to detect extrathoracic spread of lung cancer. A direct comparison between whole-body MRI and FDG-PET/CT has shown that wholebody MRI has significantly higher sensitivity for detecting metastatic disease mainly due to a higher accuracy in determining the degree of brain, neck and bone involvement ${ }^{(37-39)}$.
Contrast-enhanced perfusion MRI is a straightforward and easy-to-implement technique. Three-dimensional T1weighted gradient echo sequences with the use of parallel imaging and echo sharing allow for short acquisition times of approximately $1.5 \mathrm{~s}$ for a $3 \mathrm{D}$ dataset (so-called $4 \mathrm{D}$ or $3 \mathrm{D}$ $+t$ ) required to visualize perfusion during the peak enhancement of the lung parenchyma.

\section{CONCLUSION}

With its rapid development in recent years, MRI of the lung is now truly at the threshold of broad clinical application. Disease of the airways and vasculature, as well as nodules and lung cancer, now constitute a major focus. Because MRI offers a number of advantages when compared with conventional nuclear medicine techniques, it is competing with multidetector $\mathrm{CT}$ in many applications. The fact that it does not involve the use of ionizing radiation puts chest MRI in a front-line position for all cross-sectional imaging studies, particularly in cases involving young patients. The unique combination of structural and functional information makes MRI attractive for use in all diseases where the choices among innovative and expensive treatment options will actually require and benefit from an increased number of measurable parameters.

\section{REFERENCES}

1. Wild JM, Marshall H, Bock M, et al. MRI of the lung (1/3): methods. Insights Imaging. 2012;3:345-53.

2. Biederer J, Beer M, Hirsch W, et al. MRI of the lung (2/3). Why .. when ... how? Insights Imaging. 2012;3:355-71.

3. Koenigkam Santos M, Elias Júnior J, Mauad FM, et al. Ressonância magnética do tórax: aplicações tradicionais e novas, com ênfase em pneumologia. J Bras Pneumol. 2011;37:242-58.

4. Koyama H, Ohno Y, Kono A, et al. Quantitative and qualitative assessment of non-contrast-enhanced pulmonary MR imaging for management of pulmonary nodules in 161 subjects. Eur Radiol. 2008;18:2120-31. 
5. Hochhegger B, Marchiori E, dos Reis DQ, et al. Chemical-shift MRI of pulmonary hamartomas: initial experience using a modified technique to assess nodule fat. AJR Am J Roentgenol. 2012;199: W331-4.

6. Mori T, Nomori H, Ikeda K, et al. Diffusion-weighted magnetic resonance imaging for diagnosing malignant pulmonary nodules/ masses: comparison with positron emission tomography. J Thorac Oncol. 2008;3:358-64.

7. Hochhegger B, Marchiori E, Sedlaczek O, et al. MRI in lung cancer: a pictorial essay. Br J Radiol. 2011;84:661-8.

8. Hochhegger B, Marchiori E, Souza LS Jr, et al. Magnetic resonance in N staging of lung cancer. Eur J Radiol. 2013;82:193.

9. Ohno Y, Koyama H, Onishi Y, et al. Non-small cell lung cancer: whole-body MR examination for M-stage assessment - utility for whole-body diffusion-weighted imaging compared with integrated FDG PET/CT. Radiology. 2008;248:643-54.

10. Hochhegger B, Ley-Zaporozhan J, Marchiori E, et al. Magnetic resonance imaging findings in acute pulmonary embolism. Br J Radiol. $2011 ; 84: 282-7$.

11. Stein PD, Woodard PK, Hull RD, et al. Gadolinium-enhanced magnetic resonance angiography for detection of acute pulmonary embolism: an in-depth review. Chest. 2003;124:2324-8.

12. Oudkerk M, van Beek EJ, Wielopolski P, et al. Comparison of contrast-enhanced magnetic resonance angiography and conventional pulmonary angiography for the diagnosis of pulmonary embolism: a prospective study. Lancet. 2002;359:1643-7.

13. Kluge A, Luboldt W, Bachmann G. Acute pulmonary embolism to the subsegmental level: diagnostic accuracy of three MRI techniques compared with 16-MDCT. AJR Am J Roentgenol. 2006;187:W714.

14. Junqueira FP, Lima CMAO, Coutinho AC Jr, et al. Pulmonary arterial hypertension: an imaging review comparing MR pulmonary angiography and perfusion with multidetector CT angiography. $\mathrm{Br}$ J Radiol. 2012;85:1446-56.

15. Hochhegger B, Irion K, Marchiori E. Radiation-free method to diagnosis of pulmonary embolism. AJR Am J Roentgenol. 2013;200: W398.

16. Junqueira FP, Lima CM, Coutinho AC Jr, et al. Magnetic resonance as an alternative imaging method for the evaluation of patients with pulmonary hypertension. Eur J Radiol. 2013;82:195-6.

17. Kreitner KF, Kunz RP, Ley S, et al. Chronic thromboembolic pulmonary hypertension - assessment by magnetic resonance imaging. Eur Radiol. 2007;17:11-21.

18. Bhalla M, Turcios N, Aponte V, et al. Cystic fibrosis: scoring system with thin-section CT. Radiology. 1991;179:783-8.

19. Brody AS, Molina PL, Klein JS, et al. High-resolution computed tomography of the chest in children with cystic fibrosis: support for use as an outcome surrogate. Pediatr Radiol. 1999;29:731-5.

20. Hochhegger B, Irion KL, Marchiori E. Chest MRI in patients with cystic fibrosis: a radiation-free method. Thorax. 2013;68:105-6.

21. Levin DL, Chen Q, Zhang M, et al. Evaluation of regional pulmonary perfusion using ultrafast magnetic resonance imaging. Magn Reson Med. 2001;46:166-71.
22. Sridharan S, Derrick G, Deanfield J, et al. Assessment of differential branch pulmonary blood flow: a comparative study of phase contrast magnetic resonance imaging and radionuclide lung perfusion imaging. Heart. 2006;92:963-8.

23. Levin DL, Hatabu H. MR evaluation of pulmonary blood flow. J Thorac Imaging. 2004;19:241-9.

24. Chen Q, Mai VM, Bankier AA, et al. Ultrafast MR grid-tagging sequence for assessment of local mechanical properties of the lungs. Magn Reson Med. 2001;45:24-8.

25. Sundaram TA, Gee JC. Towards a model of lung biomechanics: pulmonary kinematics via registration of serial lung images. Med Image Anal. 2005;9:524-37.

26. Middleton H, Black RD, Saam B, et al. MR imaging with hyperpolarized 3He gas. Magn Reson Med. 1995;33:271-5.

27. Puderbach M, Eichinger M, Haeselbarth J, et al. Assessment of morphological MRI for pulmonary changes in cystic fibrosis (CF) patients: comparison to thin-section CT and chest $\mathrm{x}$-ray. Invest Radiol. 2007;42:715-25.

28. Eibel R. Pulmonary infections - Pneumonia. In: Kauczor HU, editor. MRI of the lung. Heidelberg: Springer Berlin Heidelberg; 2009. p. $255-77$.

29. Eibel R, Herzog P, Dietrich O, et al. Pulmonary abnormalities in immunocompromised patients: comparative detection with parallel acquisition MR imaging and thin-section helical CT. Radiology. 2006;241:880-91.

30. Marchiori E, Zanetti G, Rafful PP, et al. Pleural endometriosis and recurrent pneumothorax: the role of magnetic resonance imaging. Ann Thorac Surg. 2012;93:696-7.

31. Hochhegger B, Marchiori E, Souza AS Jr, et al. MRI and CT findings of metastatic pulmonary calcification. Br J Radiol. 2012;85: e69-72.

32. Hochhegger B, Marchiori E, Irion K. Acute pulmonary embolism. N Engl J Med. 2010;363:1972-5.

33. Fink C, Puderbach M, Biederer J, et al. Lung MRI at 1.5 and 3 Tesla: observer preference study and lesion contrast using five different pulse sequences. Invest Radiol. 2007;42:377-83.

34. Puderbach M, Hintze C, Ley S, et al. MR imaging of the chest: a practical approach at 1.5T. Eur J Radiol. 2007;64:345-55.

35. Ohno Y, Koyama H, Nogami M, et al. Whole-body MR imaging vs. FDG-PET: comparison of accuracy of M-stage diagnosis for lung cancer patients. J Magn Reson Imaging. 2007;26:498-509.

36. Razek AA. Diffusion magnetic resonance imaging of chest tumors. Cancer Imaging. 2012;12:452-63.

37. Hochhegger B, Marchiori E, Irion K, et al. Magnetic resonance of the lung: a step forward in the study of lung disease. J Bras Pneumol. 2012;38:105-15.

38. Hochhegger B, Irion K, Marchiori E. Whole-body magnetic resonance imaging: a viable alternative to positron emission tomography/CT in the evaluation of neoplastic diseases. J Bras Pneumol. 2010;36:396.

39. Barreto MM, Rafful PP, Rodrigues RS, et al. Correlation between computed tomographic and magnetic resonance imaging findings of parenchymal lung diseases. Eur J Radiol. 2013;82:e492-501. 